\title{
Matrix Product Steady States as Superposition of Product Shock Measures in 1D Driven Systems
}

\author{
F H Jafarpour ${ }^{1 *}$ and S R Masharian ${ }^{2}$ \\ ${ }^{1}$ Bu-Ali Sina University, Physics Department, Hamadan, Iran \\ ${ }^{2}$ Institute for Advanced Studies in Basic Sciences, Zanjan, Iran
}

October 24, 2018

\begin{abstract}
It is known that exact traveling wave solutions exist for families of $(n+1)$-states stochastic one-dimensional non-equilibrium lattice models with open boundaries provided that some constraints on the reaction rates are fulfilled. These solutions describe the diffusive motion of a product shock or a domain wall with the dynamics of a simple biased random walker. The steady state of these systems can be written in terms of linear superposition of such shocks or domain walls. These steady states can also be expressed in a matrix product form. We show that in this case the associated quadratic algebra of the system has always a twodimensional representation with a generic structure. A couple of examples for $n=1$ and $n=2$ cases are presented.
\end{abstract}

On the macroscopic level nonlinear hydrodynamic equations, such as the Burgers equation or the Fisher equation, might exhibit shocks in some cases 1, 2. On the microscopic level some stochastic non-equilibrium one-dimensional lattice models with open boundaries might also develop shocks provided that some constrained on the reaction rates are fulfilled 3, 4, 5.

In 6] exact traveling wave solutions have been obtained for three families of two-states driven-diffusive models in one-dimension with nearest-neighbors interactions. These three models are the Partially Asymmetric Simple Exclusion Process (PASEP) 7, 8, 9, the Branching-Coalescing Random Walk (BCRW) and the Asymmetric Kawasaki-Glauber Process (AKGP). By taking a Bernoulli shock distribution as initial distribution, the shock distribution in these models evolves in time into a linear combination of similar distributions with different shock positions. The time evolution of the shock position in these models is similar to the dynamics of a random walker moving on a one-dimensional lattice with reflecting boundaries [6. This property of the traveling wave solution is not limited to the two-states systems with nearest-neighbor interactions. Shocks in three-states lattice models with nearest-neighbor interactions 10, 11, 12, 13, 14 and also two-states lattice models with next nearest-neighbors interactions [15] have also been studied recently. As long as the time evolution of

\footnotetext{
${ }^{*}$ Corresponding author's e-mail:farhad@ipm.ir
} 
the product shock measure is equivalent to that of a random walker on a discrete lattice with homogeneous hopping rates in the bulk and special reflection rates at the boundary, the steady state of the model can be written as a linear superposition of these product shock measures.

The steady states of these systems, on the other hand, can be obtained using the Matrix Product Formalism (MPF) first introduced in 9 and then developed in 16] (for a recent review see [17]). According to the MPF the steady state weight of a given configuration of a two-states stochastic non-equilibrium onedimensional lattice models with open boundaries can be written as expectation value of product of non-commuting operators associated with different types of particles and vacancies in that configuration. These operators should satisfy an associative algebra. By using a matrix representation of this algebra or using the commutation relations of the operators one can in principle calculate the unnormalized steady state weights. Whether or not this algebra has a finite- or infinite-dimensional representation in not known in general; however, it is known that some quadratic algebras have finite-dimensional representations on special manifolds in their parameters space. For instance it has been shown that the quadratic algebra of PASEP has finite-dimensional representations given that one is restricted to some special values of the reaction rates through some constraints [18, 19]. Interestingly, it has been shown that the constraints for the existence of a two-dimensional representation are exactly those necessary for a single product shock measure as an initial configuration to have a simple random walk dynamics on the lattice [20]. It has also been shown that if one takes a product measure of $N-1$ consecutive product shocks, the shock positions have simple random walk dynamics provided that the constraints for the existence of an $N$-dimensional representation for the quadratic algebra of the PASEP are fulfilled. To see this one should adopt a slightly different definition of the shock measure. According to this new definition the shock is a product measure with density 1 at the shock positions and intermediate densities between these sites 20]. Hence the $N$-dimensional representations of the stationary algebra describe the stationary linear combination of such shock measures. The same is true for both the BCRW and the AKGP [6]. In fact it is known that the quadratic algebras associated with the BCRW and that of the AKGP have two-dimensional representations under some conditions on the reaction rates. Surprisingly, it has been shown that a single product measure in these systems will have a random walk dynamics under exactly the same constraints [21]. In [11 a three-states model with open boundary has been studied and it has been shown that the traveling shock solutions exist under some constraints on the reaction rates. Later a generalization of this model was introduced and studied in 13. The authors in 10 have shown that the quadratic algebra of this generalized model can be mapped into the quadratic algebra of the PASEP provided that the constraints under which the traveling shock solutions exist, are fulfilled. This means that the quadratic algebra of this generalized model has two dimensional representation under the same constraints.

Our major motivation in this paper is to investigate how the steady states of one-dimensional driven-diffusive systems with open boundaries when written in terms of linear superposition of Bernoulli shocks are related to the matrix product steady states. In this direction, we will consider those $(n+1)$-states stochastic non-equilibrium one-dimensional lattice models with open boundaries and nearest-neighbors interactions in which the time evolution equation of 
a single product shock measure is similar to the evolution equation for a biased single-particle random walk moving on a finite lattice with reflecting boundaries. Since the dynamics of the shock position, as a single-particle excitation, is simply a random walk it is easy to find the steady state probability distribution function of these systems in terms of linear superposition of single shocks. We will then show that the matrix product steady state of these systems can always be obtained using two-dimensional representations of their quadratic algebras. These matrix representations have always a generic form regardless of the details of the microscopic reactions. In order to confirm our assertion we will give a couple of example for both $n=1$ and $n=2$ cases.

In what follows we will first review very quickly the dynamics of a biased singleparticle random walk on a discrete lattice of finite length and show how the steady states probability distribution of this system can be obtained. The time evolution of the probability distribution function of any configuration of a Markovian interacting particle system $|P(t)\rangle$ is governed by a master equation which can be written as a Schrödinger like equation in imaginary time

$$
\frac{d}{d t}|P(t)\rangle=H|P(t)\rangle
$$

in which $H$ is a stochastic Hamiltonian 8. The matrix elements of the Hamiltonian are the transition rates between different configurations. For the onedimensional systems defined on a lattice of length $L$ with nearest neighbors interactions the Hamiltonian $H$ has the following general form

$$
H=\sum_{k=1}^{L-1} h_{k, k+1}+h_{1}+h_{L} .
$$

in which

$$
\begin{aligned}
h_{k, k+1} & =\mathcal{I}^{\otimes(k-1)} \otimes h \otimes \mathcal{I}^{\otimes(L-k-1)} \\
h_{1} & =h^{(l)} \otimes \mathcal{I}^{\otimes(L-1)} \\
h_{L} & =\mathcal{I}^{\otimes(L-1)} \otimes h^{(r)}
\end{aligned}
$$

For an $(n+1)$-states one-dimensional driven diffusive system, in which these states are associated with $n$ different types of particles and empty sites, $\mathcal{I}$ is an $(n+1) \times(n+1)$ identity matrix and $h$ is an $(n+1)^{2} \times(n+1)^{2}$ matrix for the bulk interactions. The two matrices $h^{(r)}$ and $h^{(l)}$ are both $(n+1) \times(n+1)$ square matrices and determine the interactions at the boundaries.

Let us assume that at $t=0$ the probability distribution function of the an $(n+1)$-states one-dimensional driven diffusive system is given by a product shock measure defined on a lattice of length $L$ as follows

$$
|k\rangle=\left(\begin{array}{c}
\rho_{1}^{(0)} \\
\rho_{1}^{(1)} \\
\vdots \\
\rho_{1}^{(n)}
\end{array}\right)^{\otimes k} \otimes\left(\begin{array}{c}
\rho_{2}^{(0)} \\
\rho_{2}^{(1)} \\
\vdots \\
\rho_{2}^{(n)}
\end{array}\right)^{\otimes L-k} \quad, \quad 0 \leq k \leq L
$$

in which the density of particles of type $i(i=1, \cdots, n)$ on the left (right) hand side of the shock position is $\rho_{1}^{(i)}\left(\rho_{2}^{(i)}\right)$. The density of the empty sites on the 
left and right hand sides of the shock position are given by $\rho_{1}^{(0)}=1-\sum_{i=1}^{n} \rho_{1}^{(i)}$ and $\rho_{2}^{(0)}=1-\sum_{i=1}^{n} \rho_{2}^{(i)}$ respectively. As can be seen every species of particles has a shock profile and the centers of shocks lie on each other. We assume that under some constraints on the reaction rates of the system the dynamics of this state $|k\rangle$, which is given by (1), is similar to the dynamics of a biased single-particle random walker on a discrete lattice with reflecting boundaries. In this case, regardless of particle type, the shock position hops to the left and right with the rates $\delta_{l}$ and $\delta_{r}$ respectively. The shock position also reflects from the boundaries. It reflects from the left boundary with the rate $\bar{\delta}_{r}$ and from the right boundary with the rate $\bar{\delta}_{l}$. The evolution of the state $|k\rangle$ is then given by

$$
\begin{aligned}
H|k\rangle & =\delta_{l}|k-1\rangle+\delta_{r}|k+1\rangle-\left(\delta_{l}+\delta_{r}\right)|k\rangle, 0<k<L \\
H|0\rangle & =\bar{\delta}_{r}|1\rangle-\bar{\delta}_{r}|0\rangle, \\
H|L\rangle & =\bar{\delta}_{l}|L-1\rangle-\bar{\delta}_{l}|L\rangle .
\end{aligned}
$$

Now one can construct a linear superposition of these single shocks to make the steady state of the system $\left|P^{*}\right\rangle$ as follows

$$
\left|P^{*}\right\rangle=\sum_{k=0}^{L} c_{k}|k\rangle
$$

so that it satisfies

$$
H\left|P^{*}\right\rangle=0 .
$$

Using (7)-(11) one can easily find the coefficients $c_{k}$ 's as

$$
\begin{aligned}
c_{k} & =\frac{\left(\delta_{r} / \delta_{l}\right)^{k}}{\left[\left(\delta_{r} / \bar{\delta}_{r}\right)+\frac{\left(\delta_{r} / \delta_{l}\right)}{1-\left(\delta_{r} / \delta_{l}\right)}\right]+\left[\left(\delta_{l} / \bar{\delta}_{l}\right)-\frac{1}{1-\left(\delta_{r} / \delta_{l}\right)}\right]\left(\delta_{r} / \delta_{l}\right)^{L}}, 0<k<L \\
c_{0} & =\frac{\left(\delta_{r} / \bar{\delta}_{r}\right)}{\left[\left(\delta_{r} / \bar{\delta}_{r}\right)+\frac{\left(\delta_{r} / \delta_{l}\right)}{1-\left(\delta_{r} / \delta_{l}\right)}\right]+\left[\left(\delta_{l} / \bar{\delta}_{l}\right)-\frac{1}{1-\left(\delta_{r} / \delta_{l}\right)}\right]\left(\delta_{r} / \delta_{l}\right)^{L}}, \\
c_{L} & =\frac{\left(\delta_{l} / \bar{\delta}_{l}\right)\left(\delta_{r} / \delta_{l}\right)^{L}}{\left[\left(\delta_{r} / \bar{\delta}_{r}\right)+\frac{\left(\delta_{r} / \delta_{l}\right)}{1-\left(\delta_{r} / \delta_{l}\right)}\right]+\left[\left(\delta_{l} / \bar{\delta}_{l}\right)-\frac{1}{1-\left(\delta_{r} / \delta_{l}\right)}\right]\left(\delta_{r} / \delta_{l}\right)^{L}} .
\end{aligned}
$$

The equation (10) gives the steady state probability distribution function of the system in terms of superposition of product shock measures. Because of the uniqueness of the steady state of our system, one should be able to find the same distribution function using other methods such as the MPF. In what follows we will briefly review the basic concepts of the MPF for an $(n+1)$-states system. According to the MPF the stationary probability distribution $\left|P^{*}\right\rangle$ for a system of length $L$ is assumed to be of the form

$$
\left|P^{*}\right\rangle=\frac{1}{Z}\left\langle W\left|\left(\begin{array}{c}
D_{0} \\
D_{1} \\
\vdots \\
D_{n}
\end{array}\right)^{\otimes L}\right| V\right\rangle
$$

in which $Z$ is a normalization factor and can be obtained easily using the normalization condition to be

$$
Z=\left\langle W\left|\left(\sum_{i=0}^{n} D_{i}\right)^{L}\right| V\right\rangle
$$


The operator $D_{0}$ stands for the presence of a vacancy and the operator $D_{i}$ $(i=1, \cdots, n)$ stands for the presence of a particle of type $i$ at each lattice site. These operators besides the vectors $\langle W|$ and $|V\rangle$ should satisfy the following algebra

$$
\begin{gathered}
h\left[\left(\begin{array}{c}
D_{0} \\
D_{1} \\
\vdots \\
D_{n}
\end{array}\right) \otimes\left(\begin{array}{c}
D_{0} \\
D_{1} \\
\vdots \\
D_{n}
\end{array}\right)\right]=\left(\begin{array}{c}
\bar{D}_{0} \\
\bar{D}_{1} \\
\vdots \\
\bar{D}_{n}
\end{array}\right) \otimes\left(\begin{array}{c}
D_{0} \\
D_{1} \\
\vdots \\
D_{n}
\end{array}\right)-\left(\begin{array}{c}
D_{0} \\
D_{1} \\
\vdots \\
D_{n}
\end{array}\right) \otimes\left(\begin{array}{c}
\bar{D}_{0} \\
\bar{D}_{1} \\
\vdots \\
\bar{D}_{1} \\
\bar{D}_{n}
\end{array}\right), h^{(r)}\left(\begin{array}{c}
D_{0} \\
D_{1} \\
\vdots \\
D_{1} \\
D_{n}
\end{array}\right)|V\rangle=\left(\begin{array}{c}
\bar{D}_{0} \\
\bar{D}_{1} \\
\vdots \\
D_{n}
\end{array}\right)|V\rangle \\
\left.\langle W| h^{(l)}\right)
\end{gathered}
$$

in which $\bar{D}_{i}$ 's are auxiliary operators [16, 17]. These relations define a quadratic algebra. Using the algebra or one of its matrix representations, one can calculate the probability of any configuration in the steady state. It is also possible to calculate the mean values of physical quantities such as the current of particles of different species or the correlation functions in the steady state.

As we mentioned above, by requiring some constraints on the reaction rates of the system the dynamics of a single shock can simply be given by a single random walk dynamics. From there one can construct the steady state of the system. We should note that the results obtained from the random walk picture should be equal to those obtained from the MPF and in fact we should have

$$
\frac{1}{Z}\left\langle W\left|\left(\begin{array}{c}
D_{0} \\
D_{1} \\
\vdots \\
D_{n}
\end{array}\right)^{\otimes L} \quad\right| V\right\rangle=\sum_{k=0}^{L} c_{k}|k\rangle
$$

Let us assume that we have a matrix representation for the operators $D_{i}$ 's and the vectors $|V\rangle$ and $\langle W|$. Finding the bra $\left\langle k^{\prime}\right|$ which is orthonormal to $|k\rangle$ i.e. $\left\langle k^{\prime} \mid k\right\rangle=\delta_{k^{\prime} k}$ helps us calculate the coefficients $c_{k}$. By defining

$$
\left|k^{\prime}\right\rangle=\left(\begin{array}{c}
x_{0} \\
x_{1} \\
\vdots \\
x_{n}
\end{array}\right)^{\otimes k^{\prime}} \otimes\left(\begin{array}{c}
y_{0} \\
y_{1} \\
\vdots \\
y_{n}
\end{array}\right)^{\otimes L-k^{\prime}}
$$

and requiring the orthonormality condition one finds that only four of $2 n+2$ parameters $\left\{x_{0}, \cdots, x_{n}, y_{0}, \cdots, y_{n}\right\}$ are independent. One can simply find a solution by taking $x_{0}=\frac{\rho_{2}}{\rho_{2}-\rho_{1}}, x_{1}=\cdots=x_{n}=\frac{-\left(1-\rho_{2}\right)}{\rho_{2}-\rho_{1}}, y_{0}=\frac{-\rho_{1}}{\rho_{2}-\rho_{1}}$ and $y_{1}=\cdots=y_{n}=\frac{\left(1-\rho_{1}\right)}{\rho_{2}-\rho_{1}}$ in which $\rho_{1}=\sum_{i=1}^{n} \rho_{1}^{(i)}$ and $\rho_{2}=\sum_{i=1}^{n} \rho_{2}^{(i)}$ are the total density of particles on the left and the right hand sides of the shock position respectively. By multiplying $\left\langle k^{\prime}\right|$ in (18) from the left one finds

$$
c_{k}=\frac{1}{Z}\left\langle W\left|G_{1}^{k} G_{2}^{L-k}\right| V\right\rangle
$$


in which

$$
G_{1}=\sum_{i=0}^{n} x_{i} D_{i} \quad \text { and } \quad G_{2}=\sum_{i=0}^{n} y_{i} D_{i} .
$$

We have found that the following two-dimensional representation

$$
\begin{aligned}
D_{0} & =\left(\begin{array}{cc}
\left(1-\sum_{i=1}^{n} \rho_{2}^{(i)}\right) & 0 \\
d_{0} & \frac{\delta_{r}}{\delta_{l}}\left(1-\sum_{i=1}^{n} \rho_{1}^{(i)}\right)
\end{array}\right), \\
D_{i} & =\left(\begin{array}{cc}
\rho_{2}^{(i)} & 0 \\
d_{i} & \frac{\delta_{r}}{\delta_{l}} \rho_{1}^{(i)}
\end{array}\right) \quad i=1, \cdots, n, \\
|V\rangle & =\left(\begin{array}{l}
v_{1} \\
v_{2}
\end{array}\right),\langle W|=\left(\begin{array}{ll}
w_{1} & w_{2}
\end{array}\right)
\end{aligned}
$$

in which $d_{0}=-\sum_{i=1}^{n} d_{i}$, uniquely generates the same coefficients $c_{k}$ for $k=$ $0, \cdots, L$ as in (12)-(14) provided that

$$
\begin{aligned}
d_{0} \frac{w_{2}}{w_{1}} & =\left(\rho_{2}-\rho_{1}\right) \frac{\left(\frac{\bar{\delta}_{r}}{\delta_{l}}\right)}{1-\left(\frac{\delta_{r}}{\delta_{l}}\right)+\left(\frac{\bar{\delta}_{r}}{\delta_{l}}\right)}, \\
d_{0} \frac{v_{1}}{v_{2}} & =\left(\rho_{1}-\rho_{2}\right) \frac{\left(\frac{\bar{\delta}_{l}}{\delta_{l}}\right)}{1-\left(\frac{\delta_{l}}{\delta_{r}}\right)+\left(\frac{\bar{\delta}_{l}}{\delta_{r}}\right)} .
\end{aligned}
$$

These two equations tell us how the reflection rates $\bar{\delta}_{r}$ and $\bar{\delta}_{l}$ are related to the components of the two vectors $|V\rangle$ and $\langle W|$. One should note that in (22), $d_{i}$ 's $(i=1, \cdots, n)$ are free parameters. Since the matrix $\sum_{i=0}^{n} D_{i}$ is diagonal in this representation, the normalization factor $Z$ in (16) can easily be calculated as

$$
Z=w_{1} v_{1}+\left(\frac{\delta_{r}}{\delta_{l}}\right)^{L} w_{2} v_{2}
$$

The matrix representations for the two matrices $G_{1}$ and $G_{2}$ are now given by

$$
G_{1}=\left(\begin{array}{cc}
0 & 0 \\
\frac{d_{0}}{\rho_{2}-\rho_{1}} & \frac{\delta_{r}}{\delta_{l}}
\end{array}\right), G_{2}=\left(\begin{array}{cc}
1 & 0 \\
\frac{-d_{0}}{\rho_{2}-\rho_{1}} & 0
\end{array}\right)
$$

with these properties

$$
G_{1}^{k}=\left(\frac{\delta_{r}}{\delta l}\right)^{k-1} G_{1}, G_{2}^{k}=G_{2}
$$

In summary whenever the quadratic algebra of an $(n+1)$-states system has a two-dimensional representation of the form (22) one can calculate the coefficients $c_{k}$ using (20) and conclude that the steady state of the system is a linear superposition of single product shock measures with random walk dynamics. In order to confirm our assertion we will give a couple of examples.

Let us first study the shocks in two-states systems which can be interpreted as particles (denoted by 1 ) and vacancies (denoted by 0 ) at each lattice site. The stochastic dynamics are defined in terms of transition rates. In this case the process is fully defined by the 12 rates $w_{i j}(i \neq j)$ where $i, j=1, \cdots, 4$. In the basis $(00,01,10,11)$ the reactions in the bulk of the system are as follows

$$
01 \rightleftharpoons 10 \quad w_{32}, w_{23}
$$




$$
\begin{aligned}
11 \rightarrow 10,01 & w_{34}, w_{24} \\
10,01 \rightarrow 11 & w_{43}, w_{42} \\
10,01 \rightarrow 00 & w_{13}, w_{12} \\
00 \rightarrow 10,01 & w_{31}, w_{21} \\
11 \rightleftharpoons 00 & w_{14}, w_{41} .
\end{aligned}
$$

For injection and extraction of particles at the boundaries we introduce the four rates $\alpha, \beta, \gamma$ and $\delta$. The reactions at the left and the right boundaries are

$$
\begin{array}{lc}
0 \rightleftharpoons 1 & \alpha, \gamma \quad \text { and } \\
0 \rightleftharpoons 1 & \delta, \beta
\end{array}
$$

respectively. As the first example we consider the PASEP. It is well known that the steady state of the PASEP can be written in terms of superposition of shocks with random walk dynamics provided that some constraints on the reaction and the boundaries rates are fulfilled. In the bulk of the lattice the particles diffuse to the left and right with the rates $w_{32}=x$ and $w_{23}=1$ respectively. For our continence we consider the injection and extraction rates for the left boundary as $(1-x) \alpha$ and $(1-x) \gamma$ and also for right boundary as $(1-x) \delta$ and $(1-x) \beta$. The associated quadratic algebra of this model can now be written as

$$
\begin{aligned}
& D_{1} D_{0}-x D_{0} D_{1}=\xi(1-x)\left(D_{0}+D_{1}\right) \\
& \langle W|\left(\alpha D_{0}-\gamma D_{1}-\xi\right)=0 \\
& \left(\beta D_{1}-\delta D_{0}-\xi\right)|V\rangle=0
\end{aligned}
$$

It is assumed that $\bar{D}_{0}=\xi(1-x) \mathcal{I}$ and $\bar{D}_{1}=-\xi(1-x) \mathcal{I}$ in which $\mathcal{I}$ is a $2 \times 2$ identity matrix. One can easily check the following two-dimensional representation satisfies (28)

$$
D_{0}=\left(\begin{array}{cc}
\left(1-\rho_{2}\right) & 0 \\
d_{0} & \frac{\delta_{r}}{\delta_{l}}\left(1-\rho_{1}\right)
\end{array}\right), D_{1}=\left(\begin{array}{cc}
\rho_{2} & 0 \\
-d_{0} & \frac{\delta_{r}}{\delta_{l}} \rho_{1}
\end{array}\right)
$$

provided that

$$
x=\frac{\rho_{1}\left(1-\rho_{2}\right)}{\rho_{2}\left(1-\rho_{1}\right)}, \frac{\delta_{r}}{\delta_{l}}=\frac{\rho_{2}\left(1-\rho_{2}\right)}{\rho_{1}\left(1-\rho_{1}\right)}
$$

and $\xi=\rho_{2}\left(1-\rho_{2}\right)$. The boundary rates together with $\rho_{1}, \rho_{2}$ and $x$ should also satisfy the following conditions

$$
\begin{aligned}
& \alpha\left(1-\rho_{1}\right)-\gamma \rho_{1}=\rho_{1}\left(1-\rho_{1}\right)(1-x) \\
& \beta \rho_{2}-\delta\left(1-\rho_{2}\right)=\rho_{2}\left(1-\rho_{2}\right)(1-x) .
\end{aligned}
$$

In this case none of the elements $v_{1}, v_{2}, w_{1}$ and $w_{2}$ in the vectors $|V\rangle$ and $\langle W|$ is zero. Finite-dimensional representations of the PASEP have widely been studied in 18, 19. It is known that the PASEP has also an $N$-dimensional representation provided that

$$
x^{1-N}=\kappa_{+}(\beta, \delta) \kappa_{+}(\alpha, \gamma)
$$

in which

$$
\kappa_{+}(u, v)=\frac{-u+v+1+\sqrt{(u-v-1)^{2}+4 u v}}{2 u} .
$$




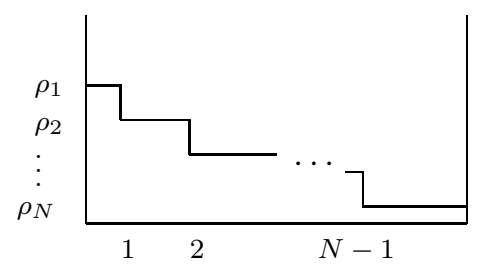

Figure 1: Sketch of a multiple shock structure in the PASEP with open boundaries. The shock positions are at the sites $1,2, \cdots$, and $N-1$.

If one takes an initial state consisting of $N-1$ consecutive shocks (see Figure 1) with the densities $\rho_{1}, \rho_{2}, \cdots$, and $\rho_{N}$, it turns out that the shock positions have simple random walk dynamics provided that the $\frac{\rho_{i+1}\left(1-\rho_{i}\right)}{\rho_{i}\left(1-\rho_{i+1}\right)}=x^{-1}$ for $i=1, \cdots, N-1$ and that

$$
\begin{gathered}
\rho_{1}=\frac{1}{1+\kappa_{+}(\alpha, \gamma)}, \\
\rho_{N}=\frac{\kappa_{+}(\beta, \delta)}{1+\kappa_{+}(\beta, \delta)} .
\end{gathered}
$$

It is interesting to note that by choosing an appropriate form for the auxiliary operators $\bar{D}_{0}$ and $\bar{D}_{1}$ an $N$-dimensional representation of the PASEP's quadratic algebra can be written as the following form

$$
\begin{aligned}
& D_{0}=\left(\begin{array}{ccccc}
\left(1-\rho_{N}\right) & 0 & \cdots & 0 & 0 \\
1 & \frac{\delta_{r, N-1}}{\delta_{l, N-1}\left(1-\rho_{N-1}\right)} & \cdots & 0 & 0 \\
0 & 1 & \cdots & 0 & 0 \\
\vdots & \vdots & \ddots & \vdots & \vdots \\
0 & 0 & \cdots & \frac{\delta_{r, N-1}}{\delta_{l, N-1}} \times \cdots \times \frac{\delta_{r, 2}}{\delta_{l, 2}}\left(1-\rho_{2}\right) & 0 \\
0 & 0 & \cdots & 1 & \frac{\delta_{r, N-1}}{\delta_{l, N-1}} \times \cdots \times \frac{\delta_{r, 1}}{\delta_{l, 1}}\left(1-\rho_{1}\right)
\end{array}\right) \\
& D_{1}=\left(\begin{array}{ccccc}
\rho_{N} & 0 & \cdots & 0 & 0 \\
0 & \frac{\delta_{r, N-1}}{\delta_{l, N-1} \rho_{N-1}} & \cdots & 0 & 0 \\
0 & 0 & \cdots & 0 & 0 \\
\vdots & \vdots & \ddots & \vdots & \vdots \\
0 & 0 & \cdots & \frac{\delta_{r, N-1}}{\delta_{l, N-1}} \times \cdots \times \frac{\delta_{r, 2}}{\delta_{l, 2}} \rho_{2} & 0 \\
0 & 0 & \cdots & 0 & \frac{\delta_{r, N-1}}{\delta_{l, N-1}} \times \cdots \times \frac{\delta_{r, 1}}{\delta_{l, 1}} \rho_{1}
\end{array}\right)
\end{aligned}
$$

in a basis in which $D_{1}$ is diagonal but not the $D_{0}$. In this matrix representation the quantities of $\delta_{r, i}$ and $\delta_{l, i}$ for $i=1, \cdots, N-1$ are the hopping rates of the $i$ th shock position to the right and to the left respectively. This can easily be done by using a similarity transformation. It is worth mentioning that this representation is exactly the one introduced in [19]; however, it is written here in terms of the densities of the shocks $\rho_{1}, \cdots, \rho_{N}$ and their hopping rates $\delta_{r, i}$ and $\delta_{l, i}$ for $i=1, \cdots, N-1$. It can be easily verified that by taking $N=2$ and applying a similarity transformation the two-dimensional representation (22) can be recovered. One should note that the eigenvalues of the matrices are not changed under similarity transformations.

As another example, we consider the BCRW as a two-states system with open boundaries and the following non-vanishing rates

$$
w_{34}, \quad w_{24}, \quad w_{42}, \quad w_{43}, \quad w_{32}, \quad w_{23}, \quad \alpha, \quad \gamma, \quad \beta
$$


and $\delta$ equal to zero. The quadratic algebra of the BCRW is

$$
\begin{aligned}
& \bar{D}_{0} D_{0}-D_{0} \bar{D}_{0}=0 \\
& \omega_{23} D_{1} D_{0}+\omega_{24} D_{1}^{2}-\left(\omega_{32}+\omega_{42}\right) D_{0} D_{1}=\bar{D}_{0} D_{1}-D_{0} \bar{D}_{1} \\
& -\left(\omega_{23}+\omega_{43}\right) D_{1} D_{0}+\omega_{34} D_{1}^{2}+\omega_{32} D_{0} D_{1}=\bar{D}_{1} D_{0}-D_{1} \bar{D}_{0} \\
& \omega_{43} D_{1} D_{0}-\left(\omega_{24}+\omega_{34}\right) D_{1}^{2}+\omega_{42} D_{0} D_{1}=\bar{D}_{1} D_{1}-D_{1} \bar{D}_{1} \\
& \langle W|\left(\alpha D_{0}-\gamma D_{1}\right)=\langle W| \bar{D}_{0}=-\langle W| \bar{D}_{1} \\
& \beta D_{1}|V\rangle=\bar{D}_{0}|V\rangle=-\bar{D}_{1}|V\rangle .
\end{aligned}
$$

It is known that for the BCRW the dynamics of a single product shock measure under the Hamiltonian of the system is a random walk provided that [6]

$$
\begin{aligned}
& \frac{1-\rho_{1}}{\rho_{1}}=\frac{\omega_{24}+\omega_{34}}{\omega_{42}+\omega_{43}} \\
& \frac{1-\rho_{1}}{\rho_{1}}=\frac{\omega_{23}}{\omega_{43}} \\
& \gamma=\frac{1-\rho_{1}}{\rho_{1}} \alpha+\left(1-\rho_{1}\right) \omega_{32}-\frac{1-\rho_{1}}{\rho_{1}} \omega_{43}+\rho_{1} \omega_{34} .
\end{aligned}
$$

In this case the density of the particles at the right hand side of the shock position is zero $\rho_{2}=0$. One can easily check that the following two-dimensional representation

$$
D_{0}=\left(\begin{array}{cc}
1 & 0 \\
d_{0} & \frac{\delta_{r}}{\delta_{l}}\left(1-\rho_{1}\right)
\end{array}\right), D_{1}=\left(\begin{array}{cc}
0 & 0 \\
-d_{0} & \frac{\delta_{r}}{\delta_{l}} \rho_{1}
\end{array}\right)
$$

with $\delta_{r}=\frac{w_{43}}{\rho_{1}}$ and $\delta_{l}=\left(1-\rho_{1}\right) w_{32}+\rho_{1} w_{34}$ satisfies (37) provided that the constraints (38) are satisfied.

For the AKGP, the non-vanishing parameters are $\omega_{12}, \omega_{13}, \omega_{42}, \omega_{43}, \omega_{32}, \alpha$ and $\beta$. The particles are allowed to enter the system only from the first site with the rate $\alpha$ and leave it from the last site of the lattice with the rate $\beta$. In this case we have $\rho_{1}=1$ and $\rho_{2}=0$. There are no additional constraints on the rates for this model. The dynamics of shock measure generated by the Hamiltonian of the system will be a simple random walk on the lattice and the shock position hopping rates are $\delta_{l}=\omega_{13}$ and $\delta_{r}=\omega_{43}$ [6]. The quadratic algebra of the AKGP is given by

$$
\begin{aligned}
& \omega_{13} D_{1} D_{0}+\omega_{12} D_{0} D_{1}=\bar{D}_{0} D_{0}-D_{0} \bar{D}_{0} \\
& -\left(\omega_{12}+\omega_{32}+\omega_{42}\right) D_{0} D_{1}=\bar{D}_{0} D_{1}-D_{0} \bar{D}_{1} \\
& -\left(\omega_{13}+\omega_{43}\right) D_{1} D_{0}+\omega_{32} D_{0} D_{1}=\bar{D}_{1} D_{0}-D_{1} \bar{D}_{0} \\
& \omega_{43} D_{1} D_{0}+\omega_{42} D_{0} D_{1}=\bar{D}_{1} D_{1}-D_{1} \bar{D}_{1} \\
& \langle W| \alpha D_{0}=\langle W| \bar{D}_{0}=-\langle W| \bar{D}_{1} \\
& \beta D_{1}|V\rangle=\bar{D}_{0}|V\rangle=-\bar{D}_{1}|V\rangle
\end{aligned}
$$

which has a two-dimensional representation given by

$$
D_{0}=\left(\begin{array}{cc}
1 & 0 \\
d_{0} & 0
\end{array}\right), D_{1}=\left(\begin{array}{cc}
0 & 0 \\
-d_{0} & \frac{\delta_{r}}{\delta_{l}}
\end{array}\right) .
$$

One can also apply our procedure to the systems with $n>2$. In what follows we investigate an open system with three-states at each lattice site associated with two different types of particles (denoted by 1 and 2) and vacancies (denoted by 0 ). The particles of different types are allowed to enter and leave the lattice from the boundaries. There is also a probability for changing the particle type at the 
boundaries. A couple of models of this type have already been studied in 10, 11, 12, 13, 14 and shown that a traveling wave solution might develop in the system. In this case the stochastic dynamics are defined in terms of 72 transition rates $w_{i j}(i \neq j)$ where $i, j=1, \cdots, 9$. In the basis $(00,01,02,10,11,12,20,21,22)$ we will consider the case studied in [14] in which the nonzero transition rates are

$$
w_{24}, w_{42}, w_{37}, w_{73}, \quad w_{86}, w_{68}, w_{61}, w_{16}, w_{18}, w_{81} .
$$

For injection and extraction of particles at the left boundary we introduce the rates :

$$
\begin{array}{ll}
1 \rightleftharpoons 0 & \alpha_{1}, \gamma_{1}, \\
1 \rightleftharpoons 2 & \alpha_{2}, \gamma_{2}, \\
0 \rightleftharpoons 2 & \alpha_{3}, \gamma_{3},
\end{array}
$$

and for the right boundary

$$
\begin{array}{ll}
1 \rightleftharpoons 0 & \delta_{1}, \beta_{1}, \\
1 \rightleftharpoons 2 & \delta_{2}, \beta_{2}, \\
0 \rightleftharpoons 2 & \delta_{3}, \beta_{3} .
\end{array}
$$

The quadratic algebra of the system in this case is given by

$$
\begin{aligned}
& -\left(w_{61}+w_{81}\right) D_{0} D_{0}+w_{16} D_{1} D_{2}+w_{18} D_{2} D_{1}=\bar{D}_{0} D_{0}-D_{0} \bar{D}_{0} \\
& -w_{42} D_{0} D_{1}+w_{24} D_{1} D_{0}=\bar{D}_{0} D_{1}-D_{0} \bar{D}_{1} \\
& -w_{73} D_{0} D_{2}+w_{37} D_{2} D_{0}=\bar{D}_{0} D_{2}-D_{0} \bar{D}_{2} \\
& w_{42} D_{0} D_{1}-w_{24} D_{1} D_{0}=\bar{D}_{1} D_{0}-D_{1} \bar{D}_{0} \\
& w_{61} D_{0} D_{0}-\left(w_{16}+w_{86}\right) D_{1} D_{2}+w_{68} D_{2} D_{1}=\bar{D}_{1} D_{2}-D_{1} \bar{D}_{2} \\
& w_{73} D_{0} D_{2}-w_{37} D_{2} D_{0}=\bar{D}_{2} D_{0}-D_{2} \bar{D}_{0} \\
& w_{81} D_{0} D_{0}+w_{86} D_{1} D_{2}-\left(w_{18}+w_{68}\right) D_{2} D_{1}=\bar{D}_{2} D_{1}-D_{2} \bar{D}_{1} \\
& \bar{D}_{2} D_{2}-D_{2} \bar{D}_{2}=\bar{D}_{1} D_{1}-D_{1} \bar{D}_{1}=0 \\
& \langle W|\left(\gamma_{1} D_{0}-\left(\alpha_{1}+\alpha_{2}\right) D_{1}+\gamma_{2} D_{2}+\bar{D}_{1}\right)=0 \\
& \langle W|\left(\alpha_{3} D_{0}+\alpha_{2} D_{1}-\left(\gamma_{2}+\gamma_{3}\right) D_{2}+\bar{D}_{2}\right)=0 \\
& \left(\beta_{1} D_{0}-\left(\delta_{1}+\delta_{2}\right) D_{1}+\beta_{2} D_{2}-\bar{D}_{1}\right)|V\rangle=0 \\
& \left(\delta_{3} D_{0}+\delta_{2} D_{1}-\left(\beta_{2}+\beta_{3}\right) D_{2}-\bar{D}_{2}\right)|V\rangle=0
\end{aligned}
$$

It has been shown that the shocks of the form (6) can develop in the system provided that some constraints are fulfilled. The shock in this model only hops to the left i.e. $\delta_{l} \neq 0$ and $\delta_{r}=0$. In the following we show that the steady state of the system can be written as a matrix product state using two-dimensional representations of the quadratic algebra (45). We will study two different cases. In the first case we assume $w_{42}=w_{18}=w_{68}=0$ and also $\rho_{2}^{(1)}=1, \rho_{2}^{(2)}=0$. The two densities $\rho_{1}^{(1)}$ and $\rho_{1}^{(2)}$ are free parameters. The matrix representation of the algebra (45) is now given by

$$
D_{0}=\left(\begin{array}{cc}
0 & 0 \\
d_{0} & 0
\end{array}\right), D_{1}=\left(\begin{array}{cc}
1 & 0 \\
d_{1} & 0
\end{array}\right), D_{2}=\left(\begin{array}{cc}
0 & 0 \\
d_{2} & 0
\end{array}\right)
$$

in which $d_{0}+d_{1}+d_{2}=0$. As can be seen the two operators $D_{0}$ and $D_{2}$ commute with each other but not necessarily with $D_{1}$. The auxiliary operators $\bar{D}_{0}, \bar{D}_{1}$ 
and $\bar{D}_{2}$ are taken to be zero. The matrix element $w_{2}$ in (22) is zero in this case and the following constraints have to be fulfilled

$$
\delta_{1}=\delta_{2}=\alpha_{1}=\alpha_{2}=0 .
$$

The rest of the boundary rates besides $d_{1}$ and $d_{2}$ should satisfy two linear equations

$$
\begin{aligned}
& \beta_{1} d_{1}+\left(\beta_{1}-\beta_{2}\right) d_{2}=0 \\
& \left(\beta_{1}+\delta_{3}\right) d_{1}+\left(\beta_{1}+\beta_{3}+\delta_{3}\right) d_{2}=0 .
\end{aligned}
$$

Assuming $d_{1}, d_{2} \neq 0$ then those boundary rates which satisfy $\beta_{1} \beta_{3}+\beta_{1} \beta_{2}+$ $\beta_{2} \delta_{3}=0$ generate a solution.

In the second case we consider $\rho_{1}^{(2)}=\rho_{2}^{(2)}=0, \rho_{2}^{(1)}=1$ and $\rho_{1}^{(1)}$ is a free parameter. The steady state of the system in this case is given by following two-dimensional matrices

$$
D_{0}=\left(\begin{array}{cc}
0 & 0 \\
d_{0} & 0
\end{array}\right), D_{1}=\left(\begin{array}{cc}
1 & 0 \\
-d_{0} & 0
\end{array}\right), D_{2}=\left(\begin{array}{ll}
0 & 0 \\
0 & 0
\end{array}\right)
$$

which is similar to (46) except that $d_{2}=0$. This means that the probability of finding second-class particles is zero in the steady state. Taking all the auxiliary operators $\bar{D}_{0}, \bar{D}_{1}$ and $\bar{D}_{2}$ equal to zero, one can simply check that (50) satisfy the algebra (45) provided that $w_{42}=0$ and

$$
\delta_{1}=\delta_{2}=\alpha_{1}=\alpha_{2}=\beta_{1}=\delta_{3}=0 .
$$

In this paper we have introduced a general procedure that can be used to investigate those one-dimensional multi-species system with open boundaries whose steady states can be written as a superposition of single product shock measures of the form (6) with random walk dynamics. Instead of applying the Hamiltonian of such systems on a single product shock measure (6) as an initial configuration and looking for the conditions under which this initial configuration evolves into a linear combination of product shock measures with different shock positions, as it is done in 11, 13, 14, 15, one can simply follow the following steps:

1. Using the standard MPF we find the quadratic algebra associated with the one-dimensional open boundaries system.

2. We investigate whether or not it has two-dimensional matrix representations and that if these representations have the same structure as (22).

3. If the answer to these questions is positive, we then calculate the coefficients $c_{k}$ 's in (10) using (20).

We should emphasis that one cannot use any arbitrary matrix representation to generate the coefficients $c_{k}$ 's using (20). Only when the two-dimensional matrix representation of the quadratic algebra is of the form (22) the coefficients $c_{k}$ 's have the right structure (12)-(14).

It would be interesting to investigate if this procedure also works for the systems with long range interactions 15] or even for the systems in which multiple product shock measures might evolve in them. As we have shown the generic 
form of the matrix representation (22) still holds for the PASEP with multiple shocks; however, it is not clear if it is true for other systems in general. It is also interesting to investigate if this property holds for the models defined a ring geometry or in the presence of second class particles. This is under our investigations.

F. H. J. would like to thank V. Rittenberg, G. M. Schütz and R. B. Stinchcombe for useful discussions and comments.

\section{References}

[1] J. M. Burgers The Non Linear Diffusion Equation (Boston: Reidel, 1974)

[2] R. A. Fisher Ann. Eugenics 7353 (1937)

[3] H. Spohn Large-Scale Dynamics of Interacting Particles (New York: Springer, 1991)

[4] F. Rezakhanlou Commun. Math. Phys. 140417 (1991)

[5] C. Kipnis and C. Landim Scaling Limits of Interacting Particle Systems (Berlin: Springer,1999)

[6] K. Krebs, F. H. Jafarpour, and G. M. Schütz New Journal of Physics 5 145.1-145.14 (2003)

[7] T. M. Liggett Stochastic Interacting Systems: Voter, Contact and Exclusion Processes (Berlin: Springer, 1999)

[8] G. M. Schütz Phase Transitions and Critical Phenomena, Vol 19, C. Domb and J. Lebowitz eds. (Academic, London, 2001)

[9] B. Derrida, M.R. Evans, V. Hakim and V. Pasquier J. Phys. A: Math. Gen. A 261493 (1993)

[10] F. H. Jafarpour and S. R. Masharian J. Stat. Mech. 03009 (2007)

[11] A. Rákos and G. M. Shcütz J. Stat. Phys. 11755 (2004)

[12] F. H. Jafarpour Physica A 358413 (2005)

[13] F. Tabatabaei, G.M. Schütz Phys. Rev. E 74051108 (2006)

[14] F. Tabatabaei, G.M. Schütz Diffusion Fundamentals 4 5.1-5.38 (2006)

[15] M. Paessens and G.M. Schütz New Journal of Physics 6120 (2004)

[16] K. Krebs and S. Sandow J. Phys. A: Math. Gen. A 303165 (1997)

[17] R. A. Blythe, M. R. Evans, arXiv:0706.1678

[18] F. H. L. Essler and V. Rittenberg J. Phys. A: Math. Gen. A 293375 (1996)

[19] K. Mallick and S. Sandow J. Phys. A: Math. Gen. A 304513 (1997)

[20] V. Belitsky and G. M. Schütz El. J. Prob. 7 Paper No.11 1 (2002)

[21] F. H. Jafarpour Physica A 339369 (2004) 